\title{
Web-Based Questionnaire for Healthcare Professionals on Psychosocial Support for Adolescents and Young Adults with Type 1 Diabetes
}

\author{
Steven James (D) - Lin Perry (D) - Christine Palmer • Margaret Harris • \\ Deepa Siram · Julia Lowe
}

Received: August 11, 2021 / Accepted: January 12, 2022 / Published online: February 1, 2022

(C) The Author(s) 2022

\begin{abstract}
Introduction: Adolescence and young adulthood is a challenging period, particularly for those living with chronic disease such as type 1 diabetes (T1D). Effective professional support is associated with better diabetes outcomes, but little is known about what determines healthcare professionals' decision-making for therapeutic intervention, and how to support this. Our study aimed to determine healthcare professionals' (HCPs) self-rated awareness, capability, opportunity and motivation to provide support for
\end{abstract}

Supplementary Information The online version contains supplementary material available at https:// doi.org/10.1007/s13300-022-01202-6.

S. James (四)

School of Nursing, Midwifery and Paramedicine, University of the Sunshine Coast, Moreton Parade, Petrie, Queensland 4502, Australia

e-mail: SJames1@usc.edu.au

L. Perry

Faulty of Health, University of Technology Sydney/

South East Sydney Local Health District, Prince of

Wales Hospital, Randwick, New South Wales 2031,

Australia

e-mail: Lin.Perry@uts.edu.au

C. Palmer · D. Siram

School of Nursing, Midwifery and Paramedicine,

University of the Sunshine Coast, Tallon Street,

Caboolture, Queensland 4510, Australia psychosocial issues in the management of T1D with adolescents and young adults; and to identify factors independently predictive of $\mathrm{HCPs}^{\prime}$ perceptions of their confidence in, and perceived importance of, addressing psychosocial issues in this population.

Methods: Survey design was used, and data collected using an anonymous web-based questionnaire based on the Capability-Opportunity-Motivation Behaviour (COM-B) framework. The study was advertised to members of the Australian Diabetes Society, and National Association of Diabetes Centres. 
Results: Of 98 respondents, 57 (58.2\%) were female. Confidence and perceived importance summary scores were not significantly associated with demographic characteristics. HCPs agreed that both diabetes-dependent and external non-diabetes-specific influences were important components of psychosocial management, but self-rated themselves as less confident in their ability to provide care for these aspects. Few respondents regularly encountered psychosocial issues that they believed would lead to improved outcomes if addressed and not all HCPs knew how to access psychosocial support for their adolescent patients.

Conclusion: Our findings indicate discrepancies between HCPs' self-rated capability and perceived motivation to provide support relating to psychosocial issues in the management of T1D for adolescents and young adults. Equitable opportunities are needed for training and support, to increase HCPs' understanding and hence their perceptions of the importance and of their confidence in addressing psychosocial issues, especially considering the high levels of risk of these young people for such problems.

Keywords: Type 1 diabetes; Young adults; Adolescence; Mental health; Healthcare professionals; Psychosocial

\section{Key Summary Points}

\section{Why carry out this study?}

Adolescence and young adulthood is recognised as a challenging period. In type 1 diabetes (T1D), effective professional support is associated with better diabetes outcomes, but little is known about what determines and how best to support healthcare professionals' (HCPs) decision-making for therapeutic intervention.

Our study determined HCPs self-rated awareness, capability, opportunity and motivation to provide psychosocial support around the management of T1D with adolescents and young adults; and sought factors independently predictive of HCPs' perceptions of their confidence in, and perceived importance of, addressing psychosocial issues in this population.

\section{What was learned from the study?}

Our findings indicate discrepancies between HCPs' self-rated capability and perceived motivation to provide psychosocial support.

Equitable opportunities are needed for training and support, to increase HCPs' understanding, and hence their perceptions of the importance and of their confidence in addressing psychosocial issues.

\section{INTRODUCTION}

Adolescence and young adulthood is a period of significant physiological and psychosocial stress, associated with the significant life and lifestyle changes that accompany passing from childhood into adulthood. These changes include moving away from parental homes to take responsibility for living independently, 
entering the workplace or further education, and dealing with relationships. Loss of familiar structures in their lives may combine with increased risk-taking behaviours, and the difficulties of this life transition are reflected in marked elevation in rates of accidents and trauma, mental health problems, alcohol and substance use. Where this transition occurs in the context of chronic disease such as diabetes, challenges multiply.

The competing challenges of becoming a mature adult can distract adolescents and young adults with type 1 diabetes (AYAT1D) from optimal disease self-management, accompanied by the health effects of uncontrolled blood glucose. Additionally, living with diabetes carries particular risks for psychosocial problems which can also be exacerbated during this period. AYAT1D may, for example, experience 'diabetes distress', which can i nclude sadness, worry, fear and anger regarding the emergence of complications, unpredictable blood glucose and hypoglycaemic episodes $[1,2]$. They may experience 'diabetes burnout', which can include strong negative feelings about their diabetes, or feeling controlled by, isolated or alone with it $[1,2]$. They might respond by avoiding diabetes management activities and be unmotivated to change this behaviour. Non-diabetes-related factors such as stressful life events, issues associated with family, community or culture, socio-economic status, literacy or health literacy and area of residence may also be influential. The consequences of this for AYAT1D include high rates of unplanned hospitalisation and premature onset of microvascular complications [3-5], which may prompt service providers to review what healthcare support is required and available for these young people.

For AYAT1D regular contact with diabetes healthcare services has been shown to support better diabetes self-management and achieve better diabetes control and outcomes [6], but attendance has often been reported as inconsistent, especially in non-metropolitan settings $[3,7]$. Key elements of this service contact include the education and support of AYAT1D provided by healthcare professionals (HCPs). However, little is known about what determines
HCPs' decision-making for therapeutic intervention and how best to support HCPs in addressing psychosocial issues when providing care for AYAT1D [8]. Such information could inform development of recommendations to better prepare HCPs to address psychosocial issues, direct continuing professional development (CPD) offerings by professional organisations and ultimately help maintain AYAT1D engagement with preventative diabetes care services.

This study aimed to determine HCPs' selfrated awareness, capability, opportunity and motivation to provide support for psychosocial issues in the management of type 1 diabetes (T1D) with adolescents and young adults. It aimed to identify factors independently predictive of HCPs' perceptions of the importance of, and their confidence in, addressing psychosocial issues in this population. For the purposes of this study, the term AYAT1D represents people with T1D aged 15-30 years. This age is recognised as a distinct phase of maturation $[9,10]$, also reflected in changes in the predominant patterns of morbidity and mortality in T1D at around 30 years of age [11].

\section{METHODS}

Survey design was used, and data were collected using an anonymous web-based questionnaire. This questionnaire was developed from the instrument used by Nichols et al. [8], in turn based on the Capability-Opportunity-Motivation Behaviour (COM-B) framework [12]. Utilised in a wide variety of healthcare-related research including, for example, medication adherence [13], this framework maps capability, opportunity and motivation interacting to generate behaviour, which in turn influences these components $[8,12]$. Capability is defined as the individual's psychological and physical capacity to engage in the activity concerned, including having the necessary knowledge or skills. Opportunity is defined as all the factors that lie outside the individual that make the behaviour possible or which prompt it $[8,12]$. Finally, motivation refers to brain processes that energise and direct behaviour, and includes 
habitual processes, emotional responses and analytical decision-making.

The tool utilised by Nichols et al. [8] comprised questions to characterize participants' age, sex, healthcare qualifications and experience. It included questions pertaining to HCPs' self-rated confidence and skills (capability) in addressing psychosocial issues in the management of T1D, the importance they perceived (motivation) to do this, and sought information on the availability and access to potential facilitators (opportunity). Finally the questionnaire comprised questions on HCPs' awareness of psychosocial management support techniques and interventions, such as motivational interviewing [14], motivational enhancement theory [15], the five 'As' [16], and the 3-min empowerment [17], choices and change [18], tipping points [19] and Flinders programs [20]. No formal validation or reliability testing had been employed.

For this study the questionnaire wording and order were modified to relate to care of AYAT1D and the response option was changed to a Likert-type rather than sliding scale, based on research team consensus. The modified questionnaire was reviewed for content validity by subject matter experts.

Principal components analysis with oblimin rotation, based on a correlation matrix with eigenvalues $>1$, examined the factor structure for questions relating to self-rated confidence. Discriminant validity was evaluated by inspecting the construct loadings of each factor, applying criteria of no cross-loading of 0.6 or more [21, 22]. Exclusion of two questions (selfrated confidence in addressing stressful life events and literacy/health literacy) resulted in a two-factor solution (Supplementary Material 1). These two factors were diabetes-dependent (perceived confidence around motivations, health beliefs, self-efficacy, behavioural habits, diabetes burdens and diabetes fears) and external non-diabetes-specific influences (perceived confidence around social support, family, community culture, socio-economic status and geographic location) (Supplementary Material 2). These factors explained $63.7 \%$ of the variance. The Kaiser-Meyer-Olkin measure of sampling adequacy was 0.83; Bartlett's tests of sphericity were significant $\left(\chi^{2}=580.49\right.$, $p<0.001)$; the diagonals of the anti-image correlation matrix were all 0.72 or above, and communalities were all 0.53 or above, excluding geographic location (0.43) and behavioural habits (0.39). Finally, Cronbach's alpha values were acceptable for both factors: diabetes-dependent (0.85), and external non-diabetesspecific influences $(0.88)$.

Similarly, principal components analysis with oblimin rotation examined the factor structure for questions relating to perceived importance. Exclusion of one question (perceived importance of literacy/health literacy) resulted in a three-factor solution (Supplementary Material 3). These three factors were: future orientation (perceived importance of motivations, health beliefs, self-efficacy and behavioural habits), psychosocial distress (perceived importance of diabetes burdens and diabetes fears), and external non-diabetes-specific influences (perceived importance of stressful life events, social support, family, community culture and socio-economic status) (Supplementary Material 2). These factors explained $62.5 \%$ of the variance. The Kaiser-Meyer-Olkin measure of sampling adequacy was 0.67; Bartlett's tests of sphericity were significant $\left(\chi^{2}=489.9\right.$, $p<0.001)$; the diagonals of the anti-image correlation matrix were all 0.52 or above (excluding perceived importance of diabetes fears $=0.448$ ), and communalities were all 0.53 or above, excluding one question relating to geographic location (0.3) which did not load onto a factor. Finally, Cronbach's alpha values were acceptable for all factors: future orientation (0.84), psychosocial distress (0.83) and external non-diabetes-specific influences (0.72).

The survey was administered through the SurveyMonkey ${ }^{\mathrm{TM}}$ platform (Supplementary Material 4). Questions were grouped on each page with the skip logic feature only presenting respondents with questions determined relevant by previous responses, reducing the risk of respondent drop-out.

The Australian Diabetes Society (ADS) advertised the study to their members via their monthly electronic newsletter for three consecutive editions. This time-period was chosen because previous similar surveys had 
demonstrated slow responses [23, 24]. The Australian National Association of Diabetes Centres (NADC) advertised the study concurrently via its bimonthly electronic newsletter for three consecutive editions, website, social media channels, and their mobile and smartphone applications with a link to the anonymous web-based questionnaire.

Eligible participants were HCPs with:

- Current or past experience as an HCP providing care to AYAT1D in Australia

- Current membership with the ADS and/or employment at a diabetes centre registered with the NADC

- Internet access

- The ability to understand and converse in the English language-expected to be met by all potential participants as it is a registration requirement for HCPs in Australia

To eliminate duplication due to overlapping recruitment through both the ADS and NADC diabetes centre routes, the questionnaire asked about previous questionnaire completion. It was not possible to determine the number of ADS or NADC members registered to receive their electronic newsletters, so all those registered to receive these electronic newsletters were targeted to maximise sample size, considering the low and slow response rates reported in previous surveys $[23,24]$. Data were collected over a period of 4 months mid-2019, and ethical approval for the study was provided by the University of the Sunshine Coast Human Research Ethics Committee (A191196). Informed consent was obtained from all participants.

Quantitative data were entered into SPSS version $24^{\mathrm{TM}}$ software. Descriptive statistics were generated and relationships between categorical variables examined using chi-square $\left(\chi^{2}\right)$ tests. Pearson's correlation determined the strength and direction of associations that exist between HCPs' perceptions of the importance and their self-rated confidence in their management of psychosocial issues in the management of T1D with adolescents and young adults. Regression analyses were also undertaken to identify associations, with dependent variables including perceived confidence and importance factor summary scores, dichotomised as $\geq$ and $<$ median values. Independent variables were dichotomised as age (18-44 vs $\geq 45$ years) and years in profession (0-5 vs $>5$ ), chosen on the basis that the views of HCPs of older vs younger age and/or more vs less experienced may differ. Additional independent variables included sex, registered nurse status, and desire to participate in formal psychosocial support training. All model assumptions were tested and met, and a $p$ value $<0.05$ was considered significant. Data from incomplete questionnaires were included in analyses where possible.

\section{RESULTS}

\section{Demographic Data}

The majority ( $n=57,58.2 \%$ ) of the 98 respondents were female. The modal group was aged $35-44$ years $(n=30,30.6 \%)$ (Table 1$)$. Most were well-educated with 91 (90.8\%) respondents having at least a bachelor's degree; 34 (34.6\%) had a masters or doctoral degree. Most were registered nurses [ $n=64,65.3 \%$-eight $(8.2 \%)$ of whom were nurse practitioners]; other respondents were predominantly medical staff $[(n=10,10.2 \%)$, general practitioners and endocrinologists $n=3,(3.1 \%)]$ and accredited practising dietitians $(n=7,7.1 \%)$. Most respondents $(n=80,81.7 \%)$ had worked in their profession for six or more years, and were currently employed ( $n=91,92.9 \%)$, working across all states and territories of Australia, although predominantly in New South Wales $(n=33,36.3 \%)$, Victoria $(n=15,16.5 \%)$ or Queensland ( $n=16,17.6 \%)$. Where reported, the employment location of $n=65(62.2 \%)$ was non-metropolitan, with regional geographical areas the commonest ( $n=38,42.2 \%$ ) work sites. Those who worked in metropolitan areas were significantly more likely to hold a higher degree $\left(51.2 \%\right.$ vs $\left.28.9 \%, \chi^{2}=4.1, p=0.04\right)$.

In an average week, $29(29.6 \%)$ respondents reported previously or currently seeing 10-20 patients with T1D, whilst $51(52 \%)$ saw fewer $(<9$ patients) and 17 (17.3\%) saw more (21-40) patients. Most $(n=82,83.7 \%)$ of these 
Table 1 Respondent characteristics

\begin{tabular}{|c|c|}
\hline & $n(\%)$ \\
\hline Male sex & $41(41.8)$ \\
\hline \multicolumn{2}{|l|}{ Age (years) } \\
\hline $18-24$ & $7(7.1)$ \\
\hline $25-34$ & $11(11.2)$ \\
\hline $35-44$ & $30(30.6)$ \\
\hline $45-54$ & $26(26.5)$ \\
\hline $55-64$ & $22(22.4)$ \\
\hline $65+$ & $2(2)$ \\
\hline \multicolumn{2}{|l|}{ Profession } \\
\hline Registered nurse & $64(65.3)$ \\
\hline Physician & $13(13.3)$ \\
\hline \multicolumn{2}{|c|}{ Experience in profession (years) } \\
\hline $0-5$ & $18(18.4)$ \\
\hline $6-15$ & $46(46.9)$ \\
\hline $16-25$ & $23(23.5)$ \\
\hline $26-35$ & $8(8.2)$ \\
\hline $36-45$ & $3(3.1)$ \\
\hline \multicolumn{2}{|c|}{ Area of employment $(n=90)$} \\
\hline Metropolitan & $25(27.8)$ \\
\hline Regional & $38(42.2)$ \\
\hline Rural & $3(3.3)$ \\
\hline Mixture & $24(26.7)$ \\
\hline
\end{tabular}

$n=98$ unless stated

respondents reported seeing $<9$ AYAT1D in the same week, but $n=13$ (13.3\%) saw 10-20 and $n=3$ (3\%) saw 21-40 AYAT1D patients.

\section{Capability and Motivation}

There were some discrepancies between HCPs' self-rated capability and perceived motivation to provide psychosocial support. Respondents largely self-rated themselves as less confident in their ability to provide care around these psychosocial aspects, but highlighted perceptions that both the T1D-dependent and external nondiabetes-specific influences were important (Table 2). Overall, there was a low to medium positive correlation between self-rated confidence and perceived importance values.

The median factor summary score for selfrated confidence was $40 ; 49(51.0 \%)$ of HCPs obtained a score greater than or equal to this value. In univariate analyses, the self-rated confidence factor summary score was not significantly associated with age (18-44 vs $\geq 45$ years), years in profession ( $0-5$ vs $>5$ ), sex, registered nurse status, or desire to participate in formal psychosocial support training. Similarly, the median factor summary score for perceived importance was also 40; 51 (53.1\%) of HCPs obtained a score greater than or equal to this value. In univariate analyses, the perceived importance factor summary score was also not significantly associated with these defined independent variables.

Considering how diagnosis and/or treatment might interact with management of the young person and their diabetes, most respondents indicated their awareness of the limitations of their expertise by seeking guidance where patients presented with, for example, eating disorders $(n=82,83.7 \%)$, psychosis $(n=80$, $81.6 \%)$, personality $(n=80,81.6 \%)$, major depressive $(n=78,79.6 \%)$ and bipolar affective disorders $(77,78.6 \%)$, schizophrenia $(n=74$, $75.5 \%)$ and substance abuse or dependence disorders $(n=71,72.4 \%)$. Around half this number indicated they would seek guidance around diabetes distress or burnout (both $n=35,35.7 \%)$. However, only two in three respondents ( $n=67,72.8 \%)$ knew where to get support if an AYAT1D presented with psychosocial issues that raised concern.

The perceived importance of addressing future orientation issues was positively associated with HCPs' desire to participate in formal psychosocial support training (beta $=0.254$, 95\% CI 0.005, 0.329, $p=0.04$ ). The perceived importance of addressing psychosocial distress and that of external diabetes features were both positively associated with self-rated confidence in addressing external diabetes features $($ beta $=$ $0.30,95 \%$ CI 0.03, 0.55, $p=0.02$; and beta $=$ 
Table 2 HCPs' perceptions of the importance and their self-rated confidence in their management of psychosocial issues

\begin{tabular}{|c|c|c|c|c|}
\hline & \multirow{2}{*}{$\begin{array}{l}\text { Confidence }^{a} \\
\text { Median (IQR) }\end{array}$} & \multirow{2}{*}{$\begin{array}{l}\text { Importance }^{\mathrm{a}} \\
\text { Median (IQR) }\end{array}$} & \multicolumn{2}{|c|}{ Pearson's correlation } \\
\hline & & & $r$ & $p$ \\
\hline \multicolumn{5}{|c|}{ T1D-dependent influences } \\
\hline Motivations & $4(4,5)$ & $5(4,5)$ & 0.313 & 0.002 \\
\hline Health beliefs & $5(4,5)$ & $5(4,5)$ & 0.351 & $<0.001$ \\
\hline Self-efficacy & $5(4,5)$ & $5(4,5)$ & 0.204 & 0.046 \\
\hline Behavioural habits & $4(3,5)$ & $5(4,5)$ & 0.019 & 0.09 \\
\hline Diabetes burdens & $4(3,5)$ & $3(3,5)$ & 0.192 & 0.06 \\
\hline Diabetes fears & $4(4,5)$ & $4(3,5)$ & -0.007 & 0.96 \\
\hline \multicolumn{5}{|c|}{ External non-diabetes-specific influences } \\
\hline Social support & $3(3,4)$ & $5(5,5)$ & 0.140 & 0.17 \\
\hline Stressful life events & & $4(3,5)$ & & \\
\hline Family & $3(3,3.75)$ & $5(4,5)$ & 0.271 & 0.01 \\
\hline Community culture & $3(3,3)$ & $4(3,5)$ & 0.306 & 0.002 \\
\hline Socio-economic status & $3(2,3)$ & $5(4,5)$ & 0.159 & 0.12 \\
\hline Geographic location & $3(3,4)$ & & & \\
\hline
\end{tabular}

$n=96$

TID type 1 diabetes

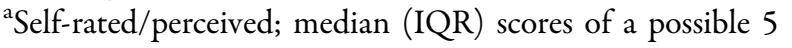

0.32, 95\% CI $\quad 0.21, \quad 0.44, \quad p=0.007$, respectively).

\section{Opportunity}

Respondents had mixed experiences of encountering in their clinical practice psychosocial issues which they believed could lead to improved outcomes, if addressed. Of 92 respondents, 14 (15.2\%) reported encountering such issues on a daily basis, whereas 21 and 20 (22.8\% and $21.7 \%$ ) reported experiencing them rarely or never, respectively. A greater proportion of HCPs who reported encountering issues on a daily basis saw more than 20 patients with T1D per week, though this difference was not statistically significant ( $n=2,66.7 \%$ vs $n=12$, 13.5\%; Fishers exact $=0.059)$. However, a significantly greater proportion were aged $\leq 44$ years $(n=12,26.1 \%$ vs $n=2,4.3 \%$;
Fishers exact $p=0.007$ ), and were not a registered nurse $(n=8,25.8 \%$ vs $n=6,9.8 \%$; $\left.\chi^{2}=4.063 ; p=0.044\right)$.

Respondents were largely aware of motivational interviewing and enhancement, but many fewer were aware of other psychosocial support programs, such as the five 'As', the 3-min empowerment, and the Flinders model (Table 3).

Of 91 respondents, more than one in three $(n=35,38.5 \%)$ respondents indicated they wanted to participate in formal psychosocial management support training. Conference sessions $(n=65,66.3 \%)$ were the most preferred learning/training medium, followed by selfguided online learning $(n=32,32.7 \%)$, regional in-person group workshops, webinar session(s) $(n=29,29.6 \%)$, and other independent study (all $n=29,29.6 \%$ ). Respondents who reported wanting to participate in formal psychosocial management support training were 
Table 3 Awareness of self-management support programs

\begin{tabular}{llll}
\hline & Not aware of & Aware of, but no resources & Aware of, had training/access to resources \\
\hline Motivational interviewing & $1(1.1)$ & $13(14.3)$ & $77(84.6)$ \\
Motivational enhancement & $15(16.5)$ & $24(24.6)$ & $52(57.1)$ \\
The 5 'As' & $33(36.3)$ & $34(37.4)$ & $24(26.4)$ \\
The 3-min empowerment & $33(36.3)$ & $22(24.2)$ & $36(36.9)$ \\
The Flinders model & $31(34.1)$ & $38(41.8)$ & $22(24.2)$ \\
\hline
\end{tabular}

$n=91$. Data are presented as number $(\%)$

significantly more likely to report encountering, on a daily, often or sometimes basis, psychosocial issues in their clinical practice that they believed would lead to improved outcomes if addressed (vs rarely or never; $53.1 \mathrm{v} 22.5 \%$, $\left.\chi^{2}=8.62, p=0.003\right)$.

To support patients' psychological and social needs, around three in four respondents $(n=72,73.5 \%)$ indicated that they had access to other HCPs with no specialised training in the psychosocial aspects of diabetes care; similar numbers $(n=71,72.4 \%)$ had access to other HCPs with such specialised training, and to a diabetes education centre $(n=78,79.6 \%)$. Five (5.1\%) respondents indicated that they had no access to any of these support mechanisms. Respondents indicated that they made use of other HCPs, both with and without specialised training in psychosocial aspects of diabetes care ( $n=34,34.7 \% ; n=52,53.1 \%$, respectively), and accessed a diabetes education centre ( $n=64,65.3 \%)$. Twelve respondents (14\%) who indicated they had access to these support mechanisms indicated that they did not make any use of them.

\section{DISCUSSION}

Our research has determined HCPs' self-rated awareness, capability, opportunity and motivation to provide support for psychosocial issues in the management of T1D with adolescents and young adults and has identified factors independently predictive of their perceptions of the importance of, and confidence in, addressing psychosocial issues in this population. Our findings indicate discrepancies between HCPs' self-rated capability and perceived motivation to provide support relating to psychosocial issues in the management of T1D for young adults. HCPs agreed that both diabetes-dependent and external non-diabetesspecific influences were important aspects of psychosocial management, but self-rated themselves as less confident in their ability to provide care for these aspects. Not all respondents reported encountering in their clinical practice psychosocial issues that they believed would lead to improved outcomes if addressed. Their reports were also mixed for their access and use of available psychosocial support. Based on the COM-B Framework, such findings will negatively influence HCPs behaviour around related psychosocial issues. There is a need for equitable opportunity around support, and to increase HCPs' understanding and hence their perceptions of the importance and their confidence in addressing psychosocial issues, especially considering the high risk levels of these young people for such problems.

Our findings are similar to those from Nichols et al. [8], and mirror discrepancies reported around diabetes healthcare support elsewhere. For example, diabetes educators have reported strongly positive intentions toward use of common diabetes-related technology for patients with T1D but also reported much lower usage [24]. Education can reduce discrepancies between HCPs' perceived motivation and selfrated capability to provide support relating to psychosocial issues for AYAT1D [25-27]. 
However, in our study only one in three respondents indicated wanting to participate in formal psychosocial management support training. Such education could, and may need to be mandated for ongoing registration or credentialing [28]. Although conferences were the preferred method of receiving education, a multimodal approach could reach more people. Greater awareness of the support available might also help. If HCPs know who they can refer to, they may be more willing to open a conversion with patients about psychosocial concerns that they currently feel unable to address. This could be achieved through formalised, consistent and regularly updated pathway documents and could involve the use of communication technologies such as videoconferencing. This would improve opportunities for support, but would require managerial sponsorship at respective locations and some resource allocation.

It was of interest to note that around one in five respondents in our study had reported either rarely or never having experiences of encountering psychosocial issues in their clinical practice which they believed could lead to improved outcomes. Even bearing in mind the varied exposure of these participants to AYAT1D (from $<9$ to up to 40 per week) this raises questions around possible lack of acknowledgement of psychosocial issues amongst the patients with whom these HCPs were working. Although speculative, this could perhaps also suggest a lack of assessment skill and interest. Our research did not seek to determine whether this was the case, and further research is warranted in this area.

Our findings should be interpreted with caution, bearing in mind the use of self-report data and potential for responder bias. As no information was available about the profile of the ADS and NADC members registered to receive their electronic newsletters, it was not possible to estimate the representativeness of the respondent profile. Our findings may be specific to the respondent group and may not relate to HCPs working with differing patient cohorts and settings. However, strengths of our research include the history and rigour of the model and questionnaire utilised, and recruitment of respondents across wide, diverse sociological and geographical areas, and healthcare disciplines, including general practitioners.

\section{CONCLUSION}

Our findings indicate discrepancies between HCPs' perceived motivation and self-rated capability to provide support relating to psychosocial issues in the management of T1D with adolescents and young adults, and inequity of opportunity and access to support resources. These findings flag the importance of finding appropriate ways to enable HCPs to provide the psychosocial support that many deem necessary yet feel underprepared to deliver. A multimodal approach may be key in supporting HCPs to align their self-rated confidence in addressing psychosocial issues with their perceptions of its importance; whilst obtaining training was clearly important to many it was not always seen as the answer. Further work is required to map preferred professional development pathways for HCPs in this area. Greater awareness of the support available may also bolster motivation, and could be achieved through formalised, consistent and regularly updated pathway documents and could involve the use of communication technologies such as video-conferencing. Such initiatives may help maximize realisation of psychosocial support in the management of T1D for adolescents and young adults.

\section{ACKNOWLEDGEMENTS}

The authors would like to thank the healthcare professionals who gave their time to the questionnaire.

Funding. Funding was granted by the University of the Sunshine Coast (Early Career Researcher Grant-Steven James). No funding or sponsorship was received for the publication of this article. 
Authorship. All named authors meet the International Committee of Medical Journal Editors (ICMJE) criteria for authorship for this article, take responsibility for the integrity of the work as a whole, and have given their approval for this version to be published.

Author Contributions. All named authors contributed to the study design and manuscript preparation. Data were collected by Steven James, and analysed by Steven James, Lin Perry and Julia Lowe.

Disclosures. All authors (Steven James, Lin Perry, Christine Palmer, Margaret Harris, Deepa Siram and Julia Lowe) have no conflicts of interest to declare.

Compliance with Ethics Guidelines. Ethical approval for the study was provided by the University of the Sunshine Coast Human Research Ethics Committee (A191196). Informed consent was obtained from all participants.

Data Availability. Data are not available, due to lack of participants' consent.

Open Access. This article is licensed under a Creative Commons Attribution-NonCommercial 4.0 International License, which permits any non-commercial use, sharing, adaptation, distribution and reproduction in any medium or format, as long as you give appropriate credit to the original author(s) and the source, provide a link to the Creative Commons licence, and indicate if changes were made. The images or other third party material in this article are included in the article's Creative Commons licence, unless indicated otherwise in a credit line to the material. If material is not included in the article's Creative Commons licence and your intended use is not permitted by statutory regulation or exceeds the permitted use, you will need to obtain permission directly from the copyright holder. To view a copy of this licence, visit http://creativecommons.org/licenses/by$\mathrm{nc} / 4.0 /$.

\section{REFERENCES}

1. American Diabetes Association. Standards of medical care in diabetes-2019. Diabetes Care. 2019;42: S91-3.

2. Craig M, Twigg S, Donaghue K, et al. National evidence-based clinical care guidelines for type 1 diabetes in children, adolescents and adults. Canberra: Australian Government; 2011.

3. Australian Institute of Health and Welfare. Diabetes among young Australians. Diabetes series no 19 Cat no CVD 59. Canberra: Australian Institute of Health and Welfare; 2012.

4. James S, Gallagher R, Dunbabin J, Perry L. Prevalence of vascular complications and factors predictive of their development in young adults with type 1 diabetes: systematic literature review. BMC Res Notes. 2014;7(593):1-11.

5. James S, Perry L, Gallagher R, et al. Service usage and vascular complications in young adults with type 1 diabetes. BMC Endoc Disord. 2014;14(39): $1-9$.

6. Monaghan $\mathrm{M}$, Helgeson $\mathrm{V}$, Wiebe D. Type 1 diabetes in young adulthood. Curr Diabetes Rev. 2015;11(4):239-50.

7. Perry L, Steinbeck K, Dunbabin J, Lowe J. Lost in transition? Access to and uptake of adult health services and outcomes for young people with type 1 diabetes in regional New South Wales. Med J Aust. 2010;193(8):444-9.

8. Nichols J, Vallis M, Boutette S, Gall Casey C, Yu C. A Canadian Cross-Sectional Survey on psychosocial supports for people living with type 1 or 2 diabetes: health-care providers' awareness, capacity, and motivation. Can J Diabetes. 2017;42:1-8.

9. Arnett J. Emerging adulthood: a theory of development from the late teens through the twenties. Am Psychol. 2000;55(5):469-80.

10. Arnett J. Emerging adulthood: the winding road from the late teens through the twenties. New York: Oxford University Press; 2004.

11. Skrivarhaug T, Bangstad H-J, Stene L. Long-term mortality in a nationwide cohort of childhood-onset type 1 diabetic patients in Norway. Diabetologia. 2006;49:298-305.

12. Michie S, van Stralen M, West R. The behaviour change wheel: a new method for characterising and designing behaviour change interventions. Implement Sci. 2011;6(42):1-11. 
13. Jackson C, Eliasson L, Barber N, Weinman J. Applying COM-B to medication adherence. A suggested framework for research and interventions. Euro Health Psychol. 2014;16(1):7-17.

14. Miller W, Rollnick S. Motivational interviewing: helping people change. New York: Guilford; 2012.

15. Miller W. Motivational enhancement therapy manual: a clinical research guide for therapists treating individuals with alcohol abuse and dependence. Collingdale: Diane; 1995.

16. Glasgow R, Emont S, Miller D. Assessing delivery of the five "As" for patient centered counseling. Health Promot Int. 2006;21:245-55.

17. Bédard J. 3-minute empowerment. 2016. http:// www.wwselfmanagement.ca/userContent/ documents/English/Professional/Resources/3_ Minutes_Empowerment.pdf. Accessed 27 Jan 2022.

18. Institute for Healthcare Communication. Choices and changes: motivating healthy behaviors. https:// healthcarecomm.org/training/faculty-courses/ choices-and-changes/. Accessed 19 May 2021.

19. Greenberg R. A new tool to motivate patients' selfcare, 2008. https://diabetesstories.com/2008/07/08/ a-new-tool-to-motivate-patients-self-care/. Accessed 19 May 2021.

20. Battersby M, Kit J, Prideaux C. Research implementing the Flinders model of self-management support with Aboriginal people who have diabetes: findings from a pilot study. Austral J Prim Health. 2008;14:66-74.
21. Matsunaga M. How to factor-analyze your data right: dos, donts and how-tos. Int J Psych Res. 2010;3(1):97-110.

22. Shaffer JA, DeGeest D, Li A. Tackling the problem of construct proliferation: A guide to assessing the discriminant validity of conceptually related constructs. Organ Res Methods. 2016;19(1):80-110.

23. Hill P, Clark R. The Australian diabetes educators' skills and readiness for the tsunami of diabetes in the 21st century. Aust J Adv Nurs. 2008;26(2):55-65.

24. James S, Perry L, Gallagher R, Lowe J. Diabetes educators' intended and reported use of common diabetes-related technologies: discrepancies and dissonance. J Diabetes Sci Tech. 2016;10(6): 1277-86.

25. Cook R, Gillespie G, Kronk R, et al. Effect of an educational intervention on nursing staff knowledge, confidence, and practice in the care of children with mild traumatic brain injury. J Neurosci Nurs. 2013;45(2):108-18.

26. Destree L, Ludwig-Beymer P, Vercellino M, Rowe A. Effects of education on nurse confidence and cardiac surgical glycemic control. AACN Adv Crit Care. 2013;24(4):362-9.

27. Greene A. What healthcare professionals can do: a view from young people with diabetes. Pediatr Diabetes. 2009;10:50-7.

28. Australian Diabetes Educators Association. Credentialling, 2020. https://www.adea.com.au/ Credentialling. Accessed 16 Jan 2020. 